\title{
DuinoBlocks: Desenho e Implementação de um Ambiente de Programação Visual para Robótica Educacional Baseado no Hardware Arduino'
}

\author{
Rafael Machado Alves, Fábio Ferrentini Sampaio \\ Instituto Tércio Pacitti de Aplicações e Pesquisas Computacionais (NCE) \\ Universidade Federal do Rio de Janeiro (UFRJ) - Cx Postal 2324 - Rio de Janeiro - RJ \\ rafamachadoalves@ufrj.br, ffs@nce.ufrj.br
}

\begin{abstract}
This paper presents a visual programming environment called DuinoBlocks developed for the Arduino robotics hardware. The DuinoBlocks is able to run on different machines with different operating systems including the PROUCA personal computers distributed by the Federal Government of Brazil to different schools. Tests conducted with the environment have shown that teachers feel more comfortable in working with this environment compared with the textual language of the Arduino (Wiring).
\end{abstract}

Resumo. Este trabalho apresenta o ambiente de programação visual DuinoBlocks desenvolvido para o hardware de robótica Arduino. O DuinoBlocks é capaz de ser executado em máquinas com diferentes sistemas operacionais, inclusive nos computadores pessoais do Programa PROUCA do Governo Federal. Os testes realizados com o ambiente têm demonstrado que professores se sentem mais confortáveis em trabalhar com esse ambiente em comparação com a linguagem textual do Arduino (Wiring).

\section{Introdução}

No futuro, os objetos do dia-a-dia terão sensores que serão capazes de se comunicar através da internet com o intuito de facilitar a vida de seus usuários na execução das tarefas do cotidiano. Esse novo paradigma de acoplar a internet a diferentes produtos é denominado Internet das Coisas [Atzori et al. 2010]. Em se tratando de cidades, uma análise dos dados coletados pelos sensores serve para tomar melhores decisões, coordenar recursos, antecipar problemas e resolvê-los de forma proativa [da Silva et al. 2012]. No Brasil, onde o assunto ainda é novidade em termos práticos, os desafios para o futuro são a busca de soluções inteligentes para a cidade lutar contra problemas recorrentes como o trânsito, diminuindo horas que são perdidas em engarrafamentos, e as enchentes, evitando tragédias causadas pelas chuvas.

Do ponto de vista do nosso grupo de pesquisa em tecnologias no ensino, a concepção e implementação - no futuro - de projetos urbanísticos inovadores para Cidades Inteligentes, implica, necessariamente, o desenvolvimento de competências e habilidades dos nossos alunos para trabalhar com inovações, robótica e outros recursos tecnológicos.

\footnotetext{
${ }^{1}$ Projeto Financiado pelo CNPq No. 550.400/2011-7
} 
$\mathrm{Na}$ atividade com Robótica Educacional (RE) o esforço do educando é empregado na criação de soluções oriundas do cotidiano dos alunos, sejam essas compostas por hardware e/ou software. As soluções visam à resolução de um problema proposto, podendo o mesmo ser real, promovendo assim a transformação do ambiente escolar em uma oficina de inventores.

É possível utilizar a robótica em sala de aula sem o uso da programação. Entretanto os projetos construídos ficam com um escopo muito limitado e, de certo modo, desconectado dos problemas reais. Desta forma, compreende-se que a inserção da programação abre a possibilidade de criação de sistemas inteligentes e autônomos capazes de reagir a um estímulo, expandindo os limites de atuação da RE.

Contudo, a linguagem de programação da maioria dos kits de robótica acessíveis às nossas Escolas são textuais, dificultando o trabalho do professor e do aluno, muitas vezes iniciantes em programação [Mendelson et al. 1990]. Por sua vez, as Linguagens de Programação Visual (ou VPL, sigla em inglês para Visual Programming Language) fornecem uma metáfora que ajuda o usuário a criar uma determinada ação (programa) com um mínimo de treinamento. Segundo Pasternak (2009), elas reduzem a carga cognitiva sobre os estudantes que aprendem sua primeira linguagem de programação.

Este trabalho promove a RE de Baixo Custo com VPL no contexto do Programa Um Computador por Aluno do Governo Federal. Trata especificamente do desenho e implementação de um ambiente de programação visual denominado DuinoBlocks, baseado na plataforma robótica Arduino (arduino.cc). Desta forma, caminha ao encontro da proposta do quarto Grande Desafio da Sociedade Brasileira de Computação [Baranauskas e Souza 2006], o qual aponta para a construção de sistemas que favoreçam o uso de tecnologias na educação por professores, melhorando a qualidade do ensino e ampliando o acesso participativo e universal do cidadão brasileiro ao conhecimento.

As próximas seções estão organizadas da seguinte forma: na Seção 2 é apresentado o contexto para o desenvolvimento do presente trabalho; em seguida, na Seção 3, encontra-se uma análise de ambientes que utilizam VPL em robótica; na Seção 4 é apresentado o contexto e os principais requisitos para o desenvolvimento do DuinoBlocks; na Seção 5 é descrito o ambiente proposto e implementado; na Seção 6 são apresentadas as avaliações do DuinoBlocks e os primeiros resultados obtidos; por fim, na Seção 7 conclui-se o artigo, tecendo as considerações finais e trabalhos futuros.

\section{Contexto de Utilização do DuinoBlocks}

O elevado custo de kits comerciais voltados para a RE ainda contribui para a pouca atividade desta no cenário da educação pública brasileira. Contudo, autores como Miranda (2010) e Santos (2010) apresentam bons resultados em se tratando de Robótica Educacional de Baixo Custo (REBC), democratizando assim o acesso às tecnologias. A REBC utiliza materiais alternativos, recursos de hardware e software livres, tais como o projeto Arduino, como forma de se viabilizar economicamente projetos na área de RE.

\subsection{O Projeto Arduino}

O projeto Arduino prevê uma plataforma de hardware e software abertos de fácil utilização, acessível não somente à especialistas na área de eletrônica, mas também 
hobbystas ou qualquer pessoa interessada na criação de objetos ou ambientes interativos. Uma vez programado, o Arduino controla uma gama de componentes eletrônicos com base em instruções recebidas através de sensores. $\mathrm{O}$ ambiente de programação nativo do Arduino - Wiring - é composto por uma linguagem baseada em comandos com características semelhantes à linguagem $\mathrm{C}$.

\subsection{O Programa PROUCA}

O Programa Um Computador por Aluno (PROUCA) é um projeto do Governo Federal com o propósito de promover a inclusão social das crianças brasileiras da rede pública de ensino mediante a aquisição de computadores portáteis novos e de baixo custo, com conteúdos pedagógicos. No final de 2011 o Ministério da Educação, através da SEED, CAPES e CNPq fazem uma chamada via Edital para que grupos de pesquisa no Brasil apresentem propostas que contemplem o uso dos laptops adquiridos pelas escolas parceiras do PROUCA [Sampaio e Elia 2012]. O projeto Uca na Cuca de pesquisa científica, pesquisa tecnológica e inovação pedagógica na área de RE é um dos selecionados pelo referido Edital.

O projeto Uca na Cuca prevê cinco metas, dentre elas a criação de um novo ambiente de desenvolvimento de programas (IDE) para o kit Arduino, incorporando uma VPL mais amigável. Esse ambiente é denominado DuinoBlocks e seus principais requisitos e características estão descritos nas Seções 4 e 5.

\section{Trabalhos Relacionados}

Foi realizada uma análise acerca dos softwares disponíveis para programação gráfica de robôs, com o intuito de comparar as suas funcionalidades equivalentes - identificando aquelas que melhor se ajustam ao contexto da proposta - bem como apontar a ausência de funcionalidades essenciais.

Dentre as VPL estudadas, duas abordagens diferentes de representação de algoritmos foram observadas. A primeira é caracterizada pela formação de empilhamentos ordenados, em que o fluxo de execução se dá de cima para baixo. Nesse grupo foram estudadas as linguagens Ardublock, Minibloq, ModKit, S4A e Squeak Etoys. A segunda abordagem é aquela em que o algoritmo tem uma estrutura de diagrama, onde o fluxo de execução segue por arestas e nós. Aqui avaliou-se as linguagens Lego Mindstorms, Microsoft Robotics Developer Studio e Firefly.

Apesar deste trabalho não visar a utilização de softwares comerciais, ainda assim, estes foram estudados como fonte de referência. Já os softwares não comerciais, além de serem parcialmente compatíveis ao contexto da proposta, permitem que desenvolvedores façam o reaproveitamento de código-fonte. Quanto à licença, os softwares tratados foram classificados da seguinte forma: Softwares não comerciais: Minibloq, Ardublock, Squeak Etoys e S4A. Softwares comerciais: ModKit, Lego Mindstorms, Firefly e Microsoft Robotics Developer Studio.

Em relação à comunicação entre o computador e o hardware Arduino, existem dois tipos de ambientes de programação. Os não autônomos, em que os projetos desenvolvidos necessitam de uma conexão constante com o computador. Em contrapartida os ambientes autônomos, solução buscada para este trabalho, permitem 
que, uma vez programado, o hardware fique independente do computador. Quanto à comunicação, os softwares de programação gráfica para o Arduino se classificam desta forma: Ambientes autônomos: Minibloq, Ardubloq e ModKit. Ambientes não autônomos: Squeak Etoys, S4A, Firefly.

A seguir serão comentados os softwares que mais influenciaram o desenvolvimento do DuinoBlocks.

MiniBLOQ (blog.minibloq.org): Um de seus principais objetivos é levar plataformas de robótica para a escola primária. Dentre suas características destacam-se: o gerador de código e a verificação de erros em tempo real; portabilidade; tradução para o português e o suporte para Linux (lançados recentemente); codificado em $\mathrm{C}++$.

ARDUBLOCK (blog.ardublock.com): Utilitário gráfico, cuja missão é gerar código compatível para o IDE Arduino que o reconhece como um "plugin". Implementa as principais funções da linguagem de programação Wiring, possui suporte multilíngue e é codificado em Java. Os blocos são representados por figuras (como no Minibloq).

S4A (seaside.citilab.eu): Uma adaptação para a robótica do Scratch, um dos ambientes de programação gráfica mais populares desenvolvido no MIT Media Lab (www.media.mit.edu). Outra adaptação do Scracth, porém não para robótica, é o BYOB (Build Your Own Blocks) que possui uma poderosa ferramenta para o usuário criar seus próprios blocos. Os blocos são representados por texto. É codificado em Smalltalk.

MoDKIT (www.modk.it): Ambiente de programação para microcontroladores que permite programar o hardware Arduino e outros compatíveis. Os blocos são inspirados no Scratch. É executado no navegador e requer um widget (ainda não disponível para Linux) na área de trabalho para se comunicar com o hardware. A detecção automática de hardware e a versão desktop foram lançadas recentemente. Sua versão gratuita não permite utilizar recursos básicos como a criação de variáveis.

Vale ressaltar que, com exceção do ModKit, nenhum dos ambientes tratados acima salvam seus projetos na nuvem.

\section{Requisitos do Sistema}

O levantamento de requisitos teve inicio na revisão da literatura e análise dos softwares que utilizam VPL (Seção 3). A equipe estendida do projeto Uca na Cuca, em diferentes seminários e reuniões, também discutiu sobre as principais características a serem implementadas no software. Por fim, as considerações feitas pelos professores que participaram dos nossos cursos de REBC (ocorridos nas escolas parceiras do Projeto), também foram de grande importância na identificação das necessidades do público alvo.

A seguir são detalhados os dois principais requisitos do ambiente DuinoBlocks:

Ambiente multiplataforma: $\mathrm{O} 1^{\circ}$ aspecto levado em consideração no desenvolvimento do DuinoBlocks foi a existência de diferentes sistemas operacionais disponíveis para alunos e professores nos diferentes equipamentos utilizados por eles. A alternativa econômica e em linha com as tendências atuais no desenvolvimento de software [Zhang et al. 2010], foi a de implementar um ambiente multiplataforma que rodasse na nuvem. 
Uma vez que o acesso à web ainda é precário em diversas escolas do Brasil e que o número de residências conectadas ainda é pequeno, pensou-se que o DuinoBlocks também pudesse rodar localmente nos navegadores das máquinas do PROUCA.

O requisito definido acima traz no seu bojo três outros importantes aspectos positivos: elimina a complexidade de uma eventual instalação, configuração ou atualização do sistema, possibilitando aos usuários o acesso ao DuinoBlocks sem a necessidade de conhecimento sobre a tecnologia utilizada; reduz a necessidade de espaço de memória ou disco nos laptops, uma vez que os projetos podem ser salvos na nuvem; e introduz facilidades para o compartilhamento e colaboração dos projetos desenvolvidos pelos alunos e professores.

Linguagem de programação amigável: Professores e alunos hoje já estão relativamente bem familiarizados com interfaces de manipulação direta como o Windows e distribuições Linux. A utilização dos recursos gráficos e mecanismos de arrastar e soltar presentes nestes ambientes é, portanto, a $1^{a}$ escolha na definição de um ambiente de programação amigável para o hardware Arduino. Desta forma, ao invés de pensarmos num ambiente tradicional (textual) de programação onde o usuário deve saber de antemão a sintaxe exata dos comandos de um programa em construção, pensou-se na utilização de blocos gráficos representando os comandos da linguagem e com um formato (visual) tal que seriam capazes de se conectar somente a alguns outros blocos de comandos de forma a evitar problemas de análise léxica e sintática.

\section{O Ambiente DuinoBlocks}

As funcionalidades do ambiente DuinoBlocks foram idealizadas levando-se em conta as necessidades relatadas na Seção 4 (Requisitos do Sistema) e outros ambientes de programação visual. Diferentes aspectos de usabilidade do ambiente foram projetados segundo referências as Leis da Simplicidade proposta por Maeda (2006). Por se tratar de um sistema com requisitos baseados em pesquisas, foi empregado no desenvolvimento do mesmo o modelo evolutivo de engenharia de software detalhado em Crinnion (1991).

A Figura 1 mostra o layout do DuinoBlocks, com um exemplo de algoritmo que faz um LED piscar. O layout é dividido em 6 painéis: o rodapé (contém botões que controlam a disposição dos painéis); o cabeçalho (barra de ferramentas com botões que realizam ações no software); o esquerdo (contém uma lista de blocos separados por categorias e subcategorias); o central (área de construção do algoritmo); o direito (contém a tradução textual do algoritmo em Wiring); e o inferior (contém os recursos necessários para carregar o programa no Arduino diretamente do navegador).

A seguir é detalhado como são abstraídas as principais estruturas lógicas de programação do ambiente DuinoBlocks.

- Categoria dos blocos. Os blocos são agrupados por categorias. Cada categoria é indicada por uma cor. Algumas categorias possuem subcategorias. Atualmente o DuinoBlocks possui seis categorias: Controle (abóbora), Operadores (verde), Entrada (roxa), Saída (azul claro), Utilitários (azul escuro) e Variáveis (vermelha).

- Criação de variáveis: para criar uma variável o usuário escolhe, na categoria Variáveis, seu nome (sem se preocupar com regras de nomeação) e seu tipo (lógica, 
numérica ou alfanumérica). Ao criar uma variável o ambiente fornece novos blocos: um para obter o seu valor e outro para alterá-lo.

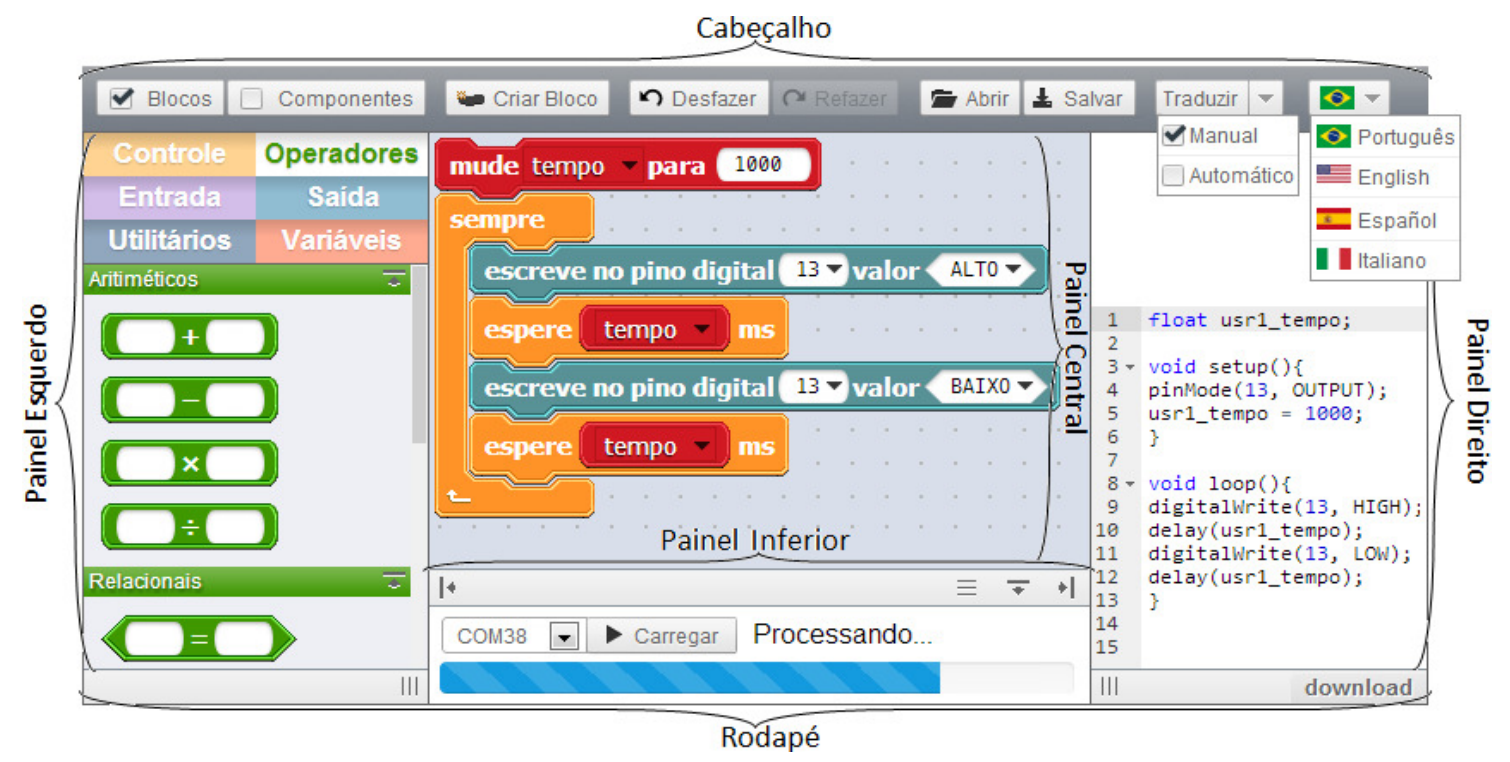

Figure 1: Layout do Ambiente DuinoBlocks

- Tipos de bloco. A forma do bloco indica os possíveis encaixes corretos sintaticamente, determinando também o seu tipo. Existem dois tipos de blocos:

- Blocos de Pilha: são passíveis de empilhamento. Possuem um entalhe no topo e uma saliência na base para serem conectados e reunidos em pilhas. Podem ser de dois subtipos diferentes. O subtipo Simples somente suporta a inserção de outros blocos de pilha no topo ou na base, já o Composto tem a forma de "C", permitindo a inserção de outros blocos de pilha, além do topo e da base, este último pode ter dois níveis de composição.

- Blocos de Retorno: são utilizados para serem encaixados dentro de outros blocos. Podem ser de três subtipos diferentes e apenas se encaixam nas cavidades de mesmo formato. O subtipo Lógico possui bordas pontiagudas e retorna um valor lógico; o Numérico possui bordas arredondadas e retorna um número; e o Alfanumérico possui bordas quadradas e retorna caracteres.

- Entrada de dados dos blocos: alguns blocos possuem entradas de dados. Estas podem ser preenchidas encaixando outros blocos, via teclado, ou escolhendo uma opção em um combobox (quando o conjunto de entradas possíveis é conhecido). O seu formato é diferente para cada tipo de entrada permitido.

- Criação de blocos pelo próprio usuário: permite a criação de procedimentos (subrotinas) pelo usuário. Para tal o usuário escolhe um nome para o procedimento, define sua categoria e tipo e edita o procedimento como se estivesse criando um novo programa. O ambiente, de forma automática, disponibiliza-o para uso no painel esquerdo.

- Módulo Componentes: apresenta um grau de abstração maior ao especializar comandos para determinados componentes (p. ex. LED, buzina, servo, motor, display de 7 segmentos, LCD, chave de contato, potenciômetro, LDR, termistor e joystick). 
O DuinoBlocks foi inicialmente concebido para programar o Arduino Uno. Porém, ele pode ser estendido para ser utilizado com qualquer versão de placa Arduino com a implementação de um módulo de "Hardwares".

Por se tratar de um ambiente com VPL, entendemos que não é coerente a exibição de mensagens de erro e o fornecimento de ajuda do ambiente serem apresentados na forma textual. Por esse motivo o DuinoBlocks possui os módulos de "Tratamento de Erro" e de "Ajuda" também na forma visual.

A escolha da tecnologia de desenvolvimento levou em consideração o esforço necessário para a programação da interface. Desta forma, optou-se pela biblioteca Pyjamas (pyjs.org), escrita em Python, e o IDE Eclipse. Outras vantagens de tais escolhas é a possibilidade de gerar código javascript automaticamente, permitindo a sua execução em qualquer navegador e rodar sem alterações como uma aplicação desktop.

Mais detalhes da arquitetura e implementação do sistema podem ser encontrados em Alves (2013).

\section{Avaliações do DuinoBlocks}

Para avaliar a proposta do DuinoBlocks e colher subsídios sobre a viabilidade de uso do ambiente, foram realizadas experimentações com o público-alvo em dois momentos. No primeiro, o DuinoBlocks foi avaliado em oficina de REBC (Subseção 6.1) e no segundo em curso de formação em RE (Subseção 6.2).

\subsection{Avaliação do DuinoBlocks em oficina de REBC}

No $1^{\circ}$ trimestre de 2013 o DuinoBlocks foi submetido a testes com o objetivo de avaliar principalmente a sua usabilidade. Foi realizada uma oficina de REBC para os alunos de um curso de Pós-Graduação em Informática na Educação, onde os participantes eram professores de diferentes áreas com idades entre 23 e 50 anos, sendo que apenas um deles tinha experiência em programação de computadores. A oficina iniciou com seis participantes e terminou com quatro, devido a mudanças na agenda de dois deles.

A oficina teve carga horária de 12 horas divididas em quatro aulas. Os participantes foram orientados a levar seus laptops pessoais e a se organizar em duplas. Cada dupla utilizou um kit de robótica. Em termos práticos, as aulas abordavam a elaboração de experimentos com montagem de componentes e a sua programação. $\mathrm{Na}$ primeira aula do curso os alunos utilizaram somente a linguagem textual do Arduino (Wiring). Nos encontros seguintes foi também incluída a linguagem gráfica do DuinoBlocks. Durante a utilização dos dois ambientes de programação, os participantes observados foram instruídos a registrar suas impressões num diário de bordo, além de responderem a questionários impressos e questionamentos orais [Cohen et al., 2005].

Uma vez que o objetivo da equipe proponente da oficina era o de testar o DuinoBlocks, não foi dada grande ênfase na montagem física dos experimentos, que por vezes foram entregues semi-prontos. Os algoritmos, por sua vez, foram, no início, elaborados de forma conjunta (professor e cursistas) e à medida que os participantes ganhavam experiência, os desafios eram propostos sem ajuda dos instrutores. 


\subsubsection{Resultados da Avaliação do DuinoBlocks em oficina de REBC}

Partimos de um processo inicialmente diretivo, onde o algoritmo (projetado com datashow) era explicado e ao mesmo tempo construído junto com os participantes que o copiavam. Num segundo momento, utilizou-se uma abordagem mais exploratória, sendo solicitadas alterações no algoritmo construído. No terceiro e último momento, trabalhou-se com plena programação.

Vale ressaltar que o nível de complexidade dos programas solicitados aos participantes desta turma foi relativamente simples. Ainda assim os resultados obtidos chamam a atençao para o fato dos mesmos serem capazes de partir de um processo de "cópia com alterações" para o de elaboração mental com o apoio do ambiente DuinoBlocks.

A fim de auxiliar o usuário no processo de encaixe de blocos decidiu-se apresentar uma área cinza, fornecendo um feedback visual para indicar uma região passível de encaixe e também emitir um som, produzindo um feedback auditivo para indicar a realização de um encaixe. Contudo, ainda se faz necessário realizar uma atualização no DuinoBlocks que modifique a forma de encaixe dos blocos, deixando esta ação mais intuitiva.

Durante a elaboração dos algoritmos percebeu-se que os participantes não tentaram fazer encaixes impossíveis, demonstrando assim, que a lógica dos blocos foi entendida. No que se refere à organização dos blocos, os agrupamentos em categorias e em subcategorias ajudaram na localização dos blocos, visto que, nenhum deles demonstrou dificuldades para encontrar um bloco representante de uma ação específica.

No último encontro da oficina (após 2 semanas de intervalo) os participantes foram solicitados a "pensar em voz alta" (técnica think aloud: [Someren et al 1994]) sobre o propósito de algoritmos apresentados em Wiring e em DuinoBlocks. Ao discursarem sobre os programas em Wiring, conseguiam relatar o objetivo de algumas partes do programa, sem - aparentemente - conseguir perceber o todo. No entanto, quando apresentados a algoritmos em DuinoBlocks, conseguiam responder corretamente sobre a funcionalidade dos mesmos, demonstrando um entendimento completo.

\subsection{Avaliação do DuinoBlocks em curso de Formação em RE}

O projeto Uca na Cuca promoveu no município de Piraí (RJ) cursos de capacitação de professores do ensino fundamental e médio intitulado "Formação em RE com Hardware Livre". Tais cursos possuíam caráter teórico-prático e a aplicação dos mesmos forneceu importantes subsídios para a especificação do DuinoBlocks.

Nas suas primeiras versões foi utilizado apenas o IDE Arduino, com sua programação textual em linguagem Wiring, para a implementação dos projetos em RE. $\mathrm{Na}$ terceira turma do curso foi introduzido o ambiente de programação visual DuinoBlocks, com o propósito de avaliar, principalmente, a sua usabilidade.

A terceira turma do curso iniciou com doze participantes, sendo cinco profissionais da área de redes de computadores com experiência em programação, um profissional de TI e seis professores de diferentes áreas, iniciantes em programação. Nas 
aulas do curso, os participantes foram organizados em grupos por bancada, sendo que os cursistas experientes em programação trabalharam juntos. Pediu-se a todos que mantivessem a mesma organização de grupos durante todos os encontros.

Nas duas aulas iniciais foram mantidas as atividades previstas no cronograma das turmas anteriores. Nelas foram abordados os seguintes assuntos: Introdução a RE, Eletrônica Básica, Apresentação do Arduino e Programação Textual Wiring. As atividades da $3^{\circ}$ aula foram alteradas e, no lugar de prosseguir com a programação Wiring, foram realizados os mesmos experimentos da aula anterior, porém com a programação em DuinoBlocks. Na última aula foram realizados novos experimentos utilizando o módulo de componentes do ambiente.

\subsubsection{Avaliação do DuinoBlocks em curso de Formação em RE}

Em comparação com as turmas anteriores, os projetos aqui executados foram construídos com uma curva de aprendizagem menor em relação ao ambiente de programação, sendo possível realizar experimentos mais complexos a partir da adoção do DuinoBlocks. Experimentos que não eram oportunos nas turmas anteriores como utilizar um display de caracteres, servo e teclado foram possíveis de serem realizados devido a abstração de complexidade dos comandos no DuinoBlocks.

Um grupo de professores chamou a atenção ao construir uma solução mais elaborada do que a esperada para a atividade de simulação do funcionamento de um semáforo de trânsito. Além dos comandos básicos esperados para a tarefa, o algoritmo construído continha um trecho responsável por fazer um LED piscar em loop. Este fato demonstrou que aqueles usuários sem conhecimento de programação foram capazes de utilizar estruturas lógicas de repetição sem a necessidade de explicações prévias.

O grupo com conhecimento em programação realizou as atividades em tempo menor que os outros e sem dificuldades. Eles utilizaram variáveis nas soluções, apesar desta funcionalidade não ter sido explicada. Para as atividades propostas o DuinoBlocks não apresentou limitações, mesmo para aqueles usuários acostumados com os ambientes textuais de programação.

\section{Conclusões e Direções Futuras}

O DuinoBlocks é um ambiente visual que estende os recursos de programação do Arduino para permitir ao iniciante, preferencialmente professores e alunos do ensino básico, programar um dispositivo robótico a fim de enriquecer o processo de ensinoaprendizagem.

A versão atual do ambiente já é capaz de rodar na nuvem, bem como na máquina do usuário com qualquer sistema operacional. Em ambas as situações o acesso ao ambiente é feito via navegador web.

Como trabalhos futuros, destacam-se: o desenvolvimento de funcionalidades que permitam salvar programas na nuvem; desenvolvimento de uma comunidade web em torno do DuinoBlocks voltada ao incentivo do compartilhamento de programas; investigar sobre as possibilidades pedagógicas do DuinoBlocks, testando-o em sala de aula com os alunos a fim de avaliar a efetividade do aprendizado de conceitos curriculares contextualizados através da robótica. 
O potencial do projeto Uca na Cuca e do DuinoBlocks, não é o de simplesmente apoiar o processo de ensino e aprendizagem, mas o de transformar o ambiente escolar em uma oficina de inventores, onde os estudantes possam trazer seus conhecimentos pessoais e interesses para a sala de aula, utilizando-os para o desenvolvimento de competências e habilidades necessárias aos cidadãos do século XXI.

\section{Referências}

Alves, R. M. DuinoBlocks: Desenho e Implementação de um Ambiente de Programação Visual para Robótica Educacional. Dissertação de Mestrado, UFRJ, RJ, 2013.

Atzori, L. Iera, A. Morabito, G. (2010) "The Internet of Things: A survey". Elsevier Computer Networks.

Baranauskas, M.C.C. e Souza, C.S. (2006) "Desafio no 4: Acesso Participativo e Universal do Cidadão Brasileiro ao Conhecimento". In: Computação Brasil, ano VII.

Cohen, L. Manion, L. Morrison, K. (2005) "Research Methods in Education”. 5th ed. Taylor \& Francis e-Library.

Crinnion, J. (1991). "Evolutionary Systems Development, a practical guide to the use of prototyping within a structured systems methodology". Plenum Press, New York.

Maeda, J. (2006) “The Laws of Simplicity”, MIT Press.

Mendelson, P.; Green, T. R. G.; Brna, P. (1990) "Programming languages in education: the search for an easy start". In Hoc, J., Green, T., Gilmore, D. \& Samway, R. (eds) Psychology of Programming, 175-200, London, Academic Press.

Miranda, L. C.; Sampaio, F. F. e Borges, J. A. dos S. (2010) "RoboFácil: Especificação e Implementação de um Kit de Robótica para a Realidade Educacional Brasileira". Em Revista Brasileira de Informática na Educação, Volume 18, Número 3.

Pasternak, E. "Visual Programming Pedagogies and Integrating Current Visual Programming Language Features". Carnegie Mellon University Robotics Institute. Thesis Master's Degree. 2009.

Sampaio e Elia (2012). "Projeto um computador por aluno: pesquisas e perspectivas". Disponível em: <http://www.nce.ufrj.br/ginape/livro-prouca>. Acesso: out de 2014.

Santos, F. L., Nascimento, F. M. S., Bezerra, R. M. S. (2010) "REDUC: A Robótica Educacional como Abordagem de Baixo Custo para o Ensino de Computação em Cursos Técnicos e Tecnológicos".

Someren, M. W. van, Barnard, Y. F., Sandberg, J. A. C. (1994) "The Think Aloud Method. A practical guide to modelling cognitive processes". Academic Press.

da Silva, V. H. S. F., Alvaro, A. (2012) "Uma Plataforma para Cidades Inteligentes baseada na Internet das Coisas", In: VIII Simpósio Brasileiro de Sistemas de Informação (SBSI), São Paulo, SP.

Zhang, S.; Zhang, S.; Chen, X.; Huo, X. (2010) "Cloud romputing research and development trend". In: International Conference on Future Networks, 2., 2010, Sanya, Hainan, China. Proceedings. Los Alamitos: IEEE. 